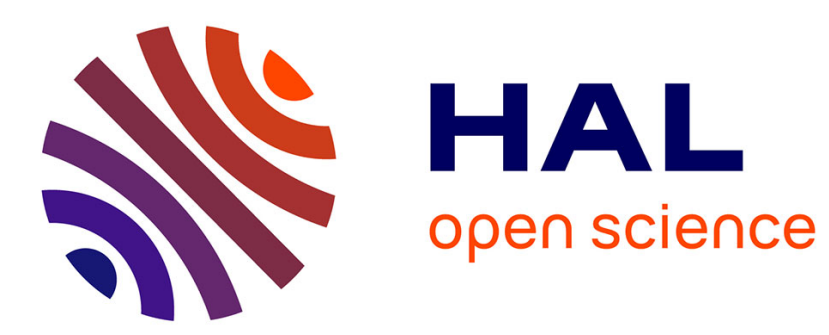

\title{
Le défi de l'égalité en contexte de développement régional au Québec
}

Denyse Côté, Tremblay-Fournier Camille

\section{To cite this version:}

Denyse Côté, Tremblay-Fournier Camille. Le défi de l'égalité en contexte de développement régional au Québec. Recherches féministes [revue interdisciplinaire francophone d'études féministes], 2011, 24 (2), pp.97-114. hal-01527868

\section{HAL Id: hal-01527868 \\ https://hal.science/hal-01527868}

Submitted on 29 May 2017

HAL is a multi-disciplinary open access archive for the deposit and dissemination of scientific research documents, whether they are published or not. The documents may come from teaching and research institutions in France or abroad, or from public or private research centers.
L'archive ouverte pluridisciplinaire HAL, est destinée au dépôt et à la diffusion de documents scientifiques de niveau recherche, publiés ou non, émanant des établissements d'enseignement et de recherche français ou étrangers, des laboratoires publics ou privés. 


\section{Le défi de l'égalité en contexte de développement régional au Québec}

\section{DENYSE CÔTÉ ET CAMILLE TREMBLAY-FOURNIER}

L'histoire du Québec a été marquée par le mouvement des femmes. Trentecinq ans après son apogée, le territoire québécois est toujours quadrillé de groupes de femmes. Leurs interventions se sont transformées au fil des ans, se muant en expertises: agressions sexuelles, violence conjugale, insertion en emploi, droits sociaux, représentation politique, entrepreneuriat au féminin, etc.

Dès ses débuts, le mouvement des femmes a dirigé ses demandes vers l'État québécois ${ }^{1}$ qui s'est peu à peu porté garant de politiques et de programmes en la matière, signant en 1997 avec le gouvernement canadien la Déclaration de Beijing. Cependant, les autorités régionales considéraient alors les groupes de femmes de leur territoire comme extérieurs à leur mission. Or le développement régional a été juxtaposé dernièrement à l'atteinte de l'égalité hommes-femmes et les tables régionales de groupes de femmes ont été associées à la structure de gouvernance et aux plans de développement régionaux (Masson 2006).

Proprement québécoise (Côté et Simard 2010), cette nouvelle configuration s'inscrit dans une tendance mondiale et soulève de nouvelles questions (Ashworth 1996), dont celle du rapport entre le mouvement féministe et les autorités régionales. Jusqu'à tout récemment aveugles au genre (Ballmer-Cao 2006), les interventions québécoises en matière de développement régional profitent-elles aux groupes de femmes? Dans quelle mesure et «comment [ceux-ci] participent-ils aux débats et aux choix démocratiques » de leur région d'appartenance (Lafontaine et Thivierge 1997 : 147)? Les groupes de femmes acquièrent-ils un nouveau pouvoir d'influence (Baron 2007) à travers cette nouvelle structuration de la démocratie et de la citoyenneté régionales (Lamoureux et Pedneault 1999)?

Ces questions nous sont apparues à la suite d'une démarche longitudinale d'observation participante auprès d'instances de gouvernance régionale au Québec et ont été confirmées par des entrevues exploratoires auprès de responsables de groupes de femmes en région. Y répondre de manière appropriée exige une analyse du contexte qui sera présentée dans la première partie de notre article. Suivra une analyse de contenu des ententes spécifiques conclues autour de 2007 dans les 17 régions du Québec, puis une analyse préliminaire du phénomène ${ }^{2}$.

1 Nous avons recours ici à l'expression «État québécois », qui fait référence, en sociologie politique québécoise, à l'appareil gouvernemental de la province de Québec.

2 À noter que nous abordons la participation des groupes de femmes en région aux processus de gouvernance régionale plutôt que la présence de femmes au sein d'instances régionales.

Recherches féministes, vol. 24, n 2, $2011: 97-114$ 
CÔTÉ ET TREMBLAY-FOURNIER $\mid 98$

\section{Les groupes de femmes en région}

Le concept de région renvoie à un découpage géographique, administratif et politique, «qui [représente] les régions comme des entités homogènes » malgré l'existence de différences notables (Côté 1995:9). La région québécoise a une « double dimension d'espace physique et politique » comme « territoire de référence du politique » (Jouve $2005: 323$ ) et comme «lieu de convergence des discours et des pratiques où institutions, secteur privé et groupes communautaires [se trouvent en situation de] proximité » (Côté 1995 : 112).

Le Québec est divisé en 17 régions administratives dont la taille varie entre celle d'une ville (Montréal ou Laval, par exemple) et d'un territoire une fois et demie plus vaste que celui de la France (Nord-du-Québec). La majorité de ces régions a connu l'effervescence des mouvements sociaux au début des années 70 , qui a provoqué l'éclatement des espaces d'intervention publique. Les gouvernements fédéral et provincial ont graduellement reconnu certaines organisations issues de ce printemps politique québécois et tissé au fil des ans des liens plus stables avec celles-ci. Le mouvement des femmes a entraîné au Québec des mutations sociales importantes : modèle familial égalitariste, nombre élevé de jeunes mères en emploi, transformation de comportements sexistes, etc. On compte toujours à l'heure actuelle plus de 500 groupes de femmes au Québec, 22 regroupements nationaux et 17 tables régionales de concertation (CDEACF 2011). Ces groupes ont choisi dès le départ l'État québécois comme interlocuteur, car il représente une cible claire, mobilisatrice, seule capable d'offrir « la reconnaissance de certains droits et l'amélioration de diverses conditions » (Laurin-Frenette $1984: 54)$.

Ces mobilisations ont aussi suscité la mise en place d'institutions intermédiaires quasi permanentes « intégrées à l'appareil étatique », tel le Conseil du statut de la femme (CSF), ce qui a permis de médiatiser «les rapports entre l'appareil gouvernemental et [...] les groupes à la base » (Laurin-Frenette 1984 : 53). Selon cette perspective, l'État devait en effet régler « la crise sociale [...] résultant de la politisation de besoins traditionnellement réservés aux sphères domestique ou économique » et créer " des liens entre les mouvements populaires et l'État à travers les discours des experts » (Fraser 1989:171). Ces institutions intermédiaires sont présentement en voie de «démantèlement ou [d]'affaiblissement» (Laurin-Frenette 1999) et remplacées, au niveau régional, par de nouveaux organismes intermédiaires régionaux : les conférences régionales des élus (CRE).

\section{Le développement et la gouvernance régionale au Québec}

Quelles sont les modalités de gouvernance et de développement au sein des régions québécoises? Répondre à cette question permettra de mieux saisir la portée 
LE DÉFI DE L'ÉGALITÉ EN CONTEXTE DE DÉVELOPPEMENT RÉGIONAL | 99

générale des transformations en cours (Peck 2004). En effet, les conditions et les pratiques développées localement sont liées entre elles (Sassen 2009) et s'articulent directement autour d'une dynamique mondiale, « à partir d'une série de flux et de réseaux » (Marques-Pereira $2000: 76$ ).

Le développement se distingue du changement social qui s'opère pour sa part de façon constante dans toute société, indépendamment des initiatives des pouvoirs publics. Le développement renvoie par contre à des pratiques volontairement axées sur la production de biens et de services dont la mise en œuvre transforme les milieux naturel, social, politique et économique (Rist 1996). Or le développement a connu des ratés, dont l'oubli des femmes n'est pas le moindre (StHilaire 1995), ratés qui ont suscité, à leur tour, une pensée sur la nécessité d'associer les femmes au développement. C'est à l'échelle de la planète que cette critique féministe « [a remis] en question les postulats [et les] théories » (Degrave $2000: 82$ ) du développement, car «les femmes ont souvent [constaté] que plus il y avait de développement, plus mauvaise devenait leur position» (Biel 2003:30). Cette critique a démontré «qu'il s'agit bien [d'un problème d]'allocation des ressources entre hommes et femmes » (Bisilliat $2000: 23$ ) et que les femmes ne sont pas un obstacle au développement.

Présentes chez les féministes des régions québécoises, ces analyses n'ont pas trouvé rapidement écho chez les responsables québécois du développement régional, puisque leur mandat se limitait initialement au développement économique et que les mandats dits sociaux, dont celui des droits des femmes, étaient plutôt assumés par le gouvernement du Québec. Les instances de gouvernance régionale ont donc conçu pendant longtemps leur mandat économique comme étranger aux mandats sociaux.

Or, dès les débuts de la décentralisation québécoise, des groupes de femmes régionaux ${ }^{3}$ avaient pourtant réclamé la dévolution d'un mandat d'égalité hommesfemmes aux instances régionales. Elles avaient "forcé » un certain investissement de l'État québécois dans des interventions localisées (Graefe 2001) : ouverture de bureaux régionaux du CSF (Tardy 2002), portraits régionaux de la situation des femmes produits par le CSF, nouvelle orientation gouvernementale en matière de condition féminine dans les régions (Gouvernement du Québec 1997a, 1997b). En 2003, les autorités régionales hériteront formellement par l'entremise d'une $1 \mathrm{loi}^{4}, \mathrm{du}$ mandat d'égalité hommes-femmes associé à certaines obligations de résultats. Cette loi substitue cependant les CRE à l'État québécois à titre d'interlocuteur des groupes régionaux. $\mathrm{Si}$ «l'écoute du gouvernement québécois aux demandes des groupes de femmes, quoique partiel, a canalisé (leur) dynamisme » (Laurin-Frenette 1984 : 54),

3 Les groupes de femmes de chaque région québécoise sont réunis au sein d'une table de concertation régionale. Ce sont les interventions de ces tables CRE, à propos des dynamiques politiques régionales auxquelles nous référons ici.

4 Un amendement de dernière minute a en effet enjoint les CRE de travailler « selon les principes de l'égalité et de la parité des femmes » (Gouvernement du Québec 2003). 
CÔTÉ ET TREMBLAY-FOURNIER $\mid 100$

dans quelle mesure les groupes de femmes régionaux seront-ils reconnus (Honneth 2008) par les CRE?

Pour répondre à cette question, il importe d'examiner la structure ainsi que la nature des rapports entre les CRE, ces organisations intermédiaires responsables de la gouvernance régionale et les groupes de femmes régionaux. Soulignons, d'entrée de jeu, que les CRE fixent le contexte d'intervention. De plus, l'approche transversale qu'elles privilégient diffère de l'approche de défense des droits des femmes portée par les groupes féministes. Dans le cadre des nouvelles modalités de gouvernance, ces derniers doivent enfin « interagir et négocier en permanence avec d'autres acteurs [locaux et régionaux] pour l'inclusion de leurs demandes » (Masson 2004 : n. p.), multiplier les démarches pour faire reconnaître leur légitimité ainsi que la particularité de leur mode d'intervention. D'autre part, les CRE recherchent des partenaires de la société civile qui pourront les appuyer dans leur mission en matière d'égalité. Pour les groupes de femmes qui participent à la concertation avec les CRE, leurs vis-à-vis, le personnel et la direction des CRE, se révèlent souvent peu versés en matière de droits des femmes.

\section{La gouvernance régionale et les groupes de femmes québécois}

La décentralisation québécoise a engendré d'importantes recompositions institutionnelles ainsi que l'imbrication de niveaux décisionnels (Brunelle 2010). Elle a suscité l'espoir d'une meilleure participation des femmes aux lieux décisionnels, car, selon ceux et celles qui en faisaient la promotion, elle devait améliorer la représentation de la population et favoriser, par la même occasion, celle des femmes qui se retrouvent habituellement en plus grand nombre à la base (Lemieux 1997). Or, on constate plutôt que, dans bien des cas, les élites locales ont été réticentes à laisser les femmes participer aux prises de décision (Ashworth 1996) et ont continué à parler au nom d'une communauté d'intérêts entre les hommes et les femmes, sans que ces dernières aient été véritablement consultées (Tardy 2002).

D'autre part, lorsque le gouvernement du Québec a confié aux CRE la responsabilité de concerter les acteurs de leur région en vue de son développement, il n'a pas formulé $d$ '" orientations claires qui refléteraient les priorités gouvernementales » (Gouvernement du Québec 2011 : 4-16) ${ }^{5}$. Or les CRE sont des entités juridiquement indépendantes, responsables directement devant leur conseil d'administration plutôt que devant la population de leur territoire. Elles ne sont qu'indirectement responsables face au gouvernement du Québec qui finance pourtant leurs activités et fixe certaines balises très larges à leur action. Leur

5 «Par exemple, la méthode retenue par le MAMROT pour répartir le FDR (Fonds de développement régional) n'est pas liée à des orientations gouvernementales, mais résulte plutôt d'un compromis émergeant de pourparlers entre les CRE »: Gouvernement du Québec (2011:4-16). 
LE DÉFI DE L'ÉGALITÉ EN CONTEXTE DE DÉVELOPPEMENT RÉGIONAL | 101

«représentation [est] non électoraliste et sélective» (Masson $2001: 26$ ). La loi prévoit que les conseils d'administration des CRE seront constitués majoritairement de préfets (nommés par les maires ou les mairesses des municipalités rurales de chaque municipalité régionale de comté $\left(\mathrm{MRC}^{6}\right)$ ) ou bien d'élus locaux (nommés par le Conseil d'une ville). Il faut noter que le nombre de conseillères et de mairesses est faible au Québec (Tardy 2002); les CRE ont donc coopté plusieurs femmes de la société civile pour contrer ce déséquilibre en matière de représentation féminine. Plusieurs CRE ont ainsi élargi la représentation de la société civile au-delà des groupes d'intérêts traditionnels (Masson 2001), mais, dans la majorité des cas (18 des $21 \mathrm{CRE}$ ), aucun groupe de femmes ne siège au conseil d'administration des CRE. Il faut plutôt se tourner vers les structures consultatives pour retrouver les groupes de femmes régionaux.

Tout comme pour d'autres structures de gouvernance (Ballmer-Cao 2006; Jouve 2005), le conseil d'administration et le personnel des CRE définissent les modalités de participation de la société civile en leur sein. Les groupes de femmes régionaux font donc face à de nouvelles règles de participation, sans avoir pu participer à leur élaboration (Brunelle 2009). Ces nouvelles structures et processus (Jouve 2005; Côté 1995; St-Hilaire 1995; Massolo 2000) imposent pourtant aux personnes qui y participent un important effort de familiarisation puisque leur mise en œuvre transforme radicalement les modalités antérieures de participation politique. Il faut en effet désormais « connaître les structures de décision et maîtriser les règles du jeu, [et] évaluer les chances et les coûts de l'intégration dans le système » (Ballmer-Cao $2006: 26$ ). Ces nouvelles règles favorisent plutôt les dirigeants d'instances décentralisées (Lebovics 2007; Tardy 2002), en particulier les élues et les élus locaux qui obtiennent un pouvoir accru en matière de gouvernance régionale, là justement où « le pouvoir est concentré » (Lamoureux et Pedneault 1999 : 4). Elles favorisent aussi les personnes de la société civile cooptées au Conseil d'administration des CRE qui, désormais, acquièrent dans bien des cas une « expertise » en matière de participation politique (Jouve 2005).

Le rôle des individus et des groupes issus de la société civile au sein des CRE est variable : observation, consultation, proposition, conciliation ou décision (Carrasco 2000). Plus précisément, le rôle des groupes de femmes régionaux au sein des CRE peut parfois consister à faire des propositions, mais il se révèle plus récemment être de l'ordre de la consultation ou de la mise en œuvre des plans d'action dressés par des ententes spécifiques. Soulignons que ces groupes doivent assumer eux-mêmes, en tout ou en grande partie, le coût de leur participation, comme c'était également le cas dans les Conseils régionaux de développement (CRD) (Minguy 2002).

Les groupes de femmes perdent ainsi la maîtrise d'œuvre de la condition féminine (Lamoureux et Pedneault 1999) dans leur région. Ils ont désormais comme

$6 \quad$ Une MRC regroupe plusieurs municipalités sur un territoire donné. 
interlocuteur privilégié un corps intermédiaire, la CRE, plutôt qu'une branche administrative, législative ou exécutive du gouvernement québécois. Les CRE assument alors le leadership régional en matière de condition féminine confié par le gouvernement du Québec en combinant des «mécanismes [de] représentation [de] groupe » [...] avec un certain souci pour la parité » (Masson $2001: 26$ ). À l'instar des anciens CRD, les CRE ne partagent pas cependant la gouvernance avec les groupes de femmes. Elles incorporent plutôt de façon sélective les demandes des groupes de femmes et les redéfinissent parfois même.

Certes, les tables de concertation régionales de groupes de femmes sont désormais des «acteurs clés issus de la société civile» (Brunelle 2009: 335) et des «partenaires» importants des plans d'action en matière d'égalité. Cependant, le type de partenariat qui leur est offert limite leur emprise sur les décisions (Brunelle 2010). Le travail de mobilisation, de conscientisation et d'appui aux femmes qui aura été celui du mouvement et des groupes de femmes récolte peu de bénéfices en matière de financement ou de pouvoir décisionnel. En ce sens, de façon générale, la décentralisation vers les paliers régionaux et l'attention que le gouvernement prête maintenant aux demandes des groupes de femmes régionaux sont loin de représenter une panacée pour ceux-ci (Lamoureux 2005; Graefe 2001).

Le développement de mécanismes au sein d'institutions décentralisées produit de «nouvelles formes de gestion sociale des femmes » qui n'encouragent pas nécessairement de «nouvelles formes d'autonomie » (St-Hilaire 1995: 36). Se pose alors la question suivante : dans quelle mesure et comment les pratiques au sein des structures de gouvernance régionale mises en place au Québec renforcent-elles la démocratie (Minguy 2002)? Les groupes de femmes arrivent-ils à préserver dans ce nouveau cadre leur autonomie ainsi qu'un certain pouvoir de négociation (Baron 2007)? Cette autonomie de la société civile est en effet nécessaire à la construction de la démocratie, même si elle s'inscrit dans le dilemme complexe de sa propre institutionnalisation (Lamoureux et Pedneault 1999 : 44-45) :

La société civile est un lieu de mobilisation et [...], à ce titre, elle conserve une autonomie vis-à-vis de l'État; elle est aussi un lieu de plus en plus important de services; à cet égard, elle devient un partenaire subalterne dans la gestion étatique des populations et des besoins sociaux.

L'amoindrissement des potentialités transformatrices des groupes de femmes à la suite de régulations trop sévères (Ballmer-Cao 2006) constitue une perte indéniable pour les sociétés ou les communautés visées. À cet effet, on constate présentement une tension nouvelle entre «le projet d'une démocratisation par la prise en charge des collectivités et celui d'une cohésion sociale à prix réduit » (Graefe $2001: 35)^{7}$. De façon générale, les instances décentralisées créent des

7 Notre traduction. 
LE DÉFI DE L'ÉGALITÉ EN CONTEXTE DE DÉVELOPPEMENT RÉGIONAL | 103

partenariats avec des associations prêtes à « jouer le jeu », car les CRE opèrent une sélection des associations appelées à participer à la concertation régionale (Jouve $2005: 336)$ : «Reste à savoir précisément comment ces instances politiques réagissent [aux] revendications [des associations]. Pourquoi et comment certains groupes accèdent, et d'autres non, à ces instances? »

Bien sûr, le fait d'avoir réussi à faire incorporer leurs demandes au sein des dispositifs de gouvernance constitue en soi une victoire importante pour le mouvement féministe. En effet, les politiques sensibles au genre sont souvent le résultat d'efforts déployés par des féministes et des progressistes (Rathgeber 1995). Cependant, le prix à payer aura-t-il été celui de la «neutralisation des voix les plus critiques » (Falquet $2008: 96$ )?

\section{L'espace politique et les groupes de femmes en région : quelques indices}

Les résultats préliminaires d'une recherche en cours viennent confirmer ces tendances. Nous avons analysé le contenu des 21 ententes spécifiques en matière d'égalité entre les femmes et les hommes en vigueur en 2011 dans les 17 régions administratives du Québec ${ }^{8}$. Ces données ne permettent certes pas de capturer les jeux et les enjeux subtils de négociation, mais elles aident à dessiner le portrait du compromis final, traduit en termes administratifs.

Apparues en 1992, généralisées dix ans plus tard, les ententes spécifiques sont des conventions établies entre différents ministères, organismes paragouvernementaux et organisations de la société civile permettant d'articuler les priorités régionales et d'adapter les normes et les priorités gouvernementales aux particularités régionales dans un domaine spécifique. Ces « engagements contractuels » formalisent le processus de concertation entre les différents acteurs et déterminent le partage des responsabilités, des ressources et des tâches entre les partenaires retenus (Bourque 2009). Elles permettent la mise en œuvre de la planification stratégique quinquennale de chaque CRE. Encadrées par le ministère de la Culture, des Communications et de la Condition féminine (MCCCF), les ententes spécifiques en matière d'égalité entre les femmes et les hommes font l'objet d'un processus de négociation instauré par chaque CRE avec un ou plusieurs ministères ainsi que des partenaires de la société civile régionale. Dans une région, le partenariat d'une entente spécifique est conclu entre les directions régionales des ministères, la CRE ainsi que, le cas échéant, certains groupes représentant la société civile aptes à contribuer financièrement à l'entente. Il s'agit là d'un partenariat « gouvernement-région », liant une région au gouvernement central.

8 Cinq ententes spécifiques ont débuté en 2008 plutôt qu'en 2007; une entente ne se termine qu'en 2013. 
Les ententes spécifiques se concluent dans des domaines variés : immigration, culture, pauvreté, par exemple. En matière de condition féminine, les premières ententes spécifiques sont apparues il y a dix ans sur une base volontaire et aléatoire ${ }^{9}$. La génération actuelle des ententes spécifiques en matière d'égalité est la première à être implantée dans toutes les régions du Québec. Les tables de concertation régionales de groupes de femmes sont appelées systématiquement à y participer. Pour elles, il s'agit souvent d'une condition d'existence, car ces ententes spécifiques leur procurent reconnaissance et financement (Bourque 2009). Toutefois, dans quelle mesure ces ententes spécifiques permettent-elles la mise en œuvre des priorités propres aux groupes de femmes (Bourque 2009)?

Les ententes spécifiques semblaient au départ ouvrir de nouveaux espaces délibératifs avec les autorités régionales. C'est ce qui a motivé la participation initiale de plusieurs groupes de femmes. Cependant, ces ententes ont été structurées selon des règles précises qui orientent la nature des interventions et opèrent un tri dans les discours ainsi que les propositions reçues et financées par les CRE. Par la force des choses, ces ententes spécifiques orientent fortement l'action des groupes de femmes régionaux et encadrent les rapports officiels et officieux entre ces groupes et les agents de la CRE : ce sont de nouvelles « règles publiques négociées » qui transforment radicalement la gestion du champ des droits des femmes dans chaque région. En effet, la mise en place d'ententes spécifiques s'accompagne habituellement de " conventions informelles [fondées] sur la confiance et [à travers lesquelles] les acteurs publics s'efforcent, à tous les niveaux, mais surtout au niveau local, d'amener des partenaires privés, notamment associatifs, à collaborer à la mise en œuvre de l'action publique » (Chevalier 2003 : 215).

Les ententes spécifiques nécessitent d'importants investissements en temps de la part des organismes gouvernementaux et non gouvernementaux conviés à la table de négociation, avant et après la signature des ententes. Dans un rapport récent, le Vérificateur général du Québec affirme à cet effet que la lourdeur administrative et de longs délais «caractérisent la conclusion des ententes » spécifiques. Dans le cas de 25 ententes spécifiques retenues à titre d'échantillon, il cite l'exemple suivant: celui d'un délai d'un an en moyenne entre l'engagement des sommes prévues par la CRE et la signature de l'entente et délais supérieurs à 600 jours dans $20 \%$ des cas retenus. Il conclut qu' " il s'agit d'un processus discutable, compte tenu des sommes en cause » (Gouvernement du Québec 2011 : 4-25).

Nous avons procédé à une analyse de contenu des ententes spécifiques triennales conclues dans le domaine de l'égalité hommes-femmes en vigueur en 2010 : titre, contenu des volets et signataires retenus sont les données à notre

9 La première génération comprenait des ententes aussi bien administratives que spécifiques. Par la suite, le MCCCF a imposé le modèle de l'entente spécifique. Pour les besoins de l'analyse, nous avons regroupé les deux types d'ententes sous le vocable « entente spécifique ». 
LE DÉFI DE L'ÉGALITÉ EN CONTEXTE DE DÉVELOPPEMENT RÉGIONAL | 105

disposition, les plans d'action des CRE étant difficiles d'accès et des entrevues avec les personnes visées étant prévues dans une prochaine étape de la recherche.

Les titres des ententes spécifiques reflètent dans la majorité des cas la terminologie gouvernementale de l'heure, soit celle de l' « égalité hommes-femmes » (13 ententes); cinq ententes ont par contre choisi la terminologie privilégiée par le mouvement des femmes, celle de condition féminine $(5 \text { ententes })^{10}$. Deux ententes font exception en privilégiant une terminologie insensible au genre: le «développement régional» (1 entente) ou la «revitalisation urbaine intégrée (RUI) » (1 entente).

Le nombre de signataires de ces ententes varie considérablement. Sauf exception, le signataire d'une entente doit y contribuer des fonds en argent sonnant (et non pas en "nature»), et la CRE doit approuver ce signataire. Les signataires forment par la suite un "comité de gestion» qui présente au conseil d'administration de la CRE des recommandations pour une décision sans appel quant au financement de projets. Un comité de suivi du plan d'action de l'entente spécifique est également mis sur pied, réunissant les partenaires «terrain » à la mise en œuvre de l'entente.

Nous avons recensé en moyenne 6,6 signataires par entente, variant entre 2 signataires dans la région de la Capitale-Nationale et 15 dans la région du Centre-duQuébec. Le MCCCF ainsi que la CRE de chaque territoire ${ }^{11}$ sont signataires de toutes les ententes et le ministère des Affaires municipales, des Régions et de l'Occupation du territoire (MAMROT) est signataire de 17 de ces 21 ententes. Le CSF a signé 8 ententes, le ou la ministre responsable de la région visée a signé 11 ententes; le ministère du Développement Économique, de l'Innovation et de l'Exportation (MDEIE), 4 ententes; le ministère de l'Emploi et de la Solidarité sociale (MESS), 1 entente. Les Forums-jeunesse ${ }^{12}$ (6 ententes), des organismes jeunesse ( 1 entente), des établissements scolaires ( 1 entente) et des commissions scolaires (3 ententes) sont aussi signataires de certaines ententes. On remarque cependant que les tables régionales de concertation des groupes de femmes ne sont signataires que du tiers des ententes spécifiques et ne représentent que $5 \%$ des

10 Le gouvernement du Québec a remplacé depuis 2005 l'expression « condition féminine », utilisée antérieurement, par celle d' «égalité entre les femmes et les hommes », ce que le mouvement féministe a interprété comme un recul.

11 Deux régions (soit le Nord-du-Québec et la Montérégie) comptent respectivement trois CRE. Chaque CRE a signé une entente spécifique ou une entente administrative.

12 Les forums jeunesse régionaux sont des tables de concertation liées à la CRE de chaque région et financées par le Secrétariat à la jeunesse dans le contexte de la Stratégie d'action jeunesse 2009-2014 du gouvernement du Québec. Ils détiennent un financement au moins vingt fois supérieur à celui des tables de concertation des groupes de femmes et reçoivent des sommes importantes (plus d'un million de dollars) pour développer un volet « égalité » dans lequel ils n'ont actuellement aucune expertise. Le mouvement des femmes a protesté en vain contre cette situation (Dutrisac 2011). 
signataires. Il en va de même des groupes de femmes œuvrant dans les secteurs de l'emploi, de la production économique, de l'entrepreneuriat et de la représentation politique qui ne forment que $9 \%$ des signataires.

Les ententes spécifiques en matière d'égalité comprennent de 2 à 9 volets, la moyenne se situant à 5,4. Les thèmes les plus récurrents sont : la participation des femmes aux instances (15), le rapport des femmes à l'économie ou au marché du travail (dont l'entrepreneuriat au féminin (9)), l'égalité économique des femmes (6) ou la diversification des emplois (7) et la mise en œuvre de l'analyse différenciée selon les sexes (ADS) $(11)^{13}$.

Les ententes où les groupes de femmes régionaux ne sont pas signataires se limitent habituellement à ces volets. Cependant, lorsqu'un groupe de femmes ou une table de concertation régionale est signataire de l'entente spécifique, celle-ci contient souvent des volets supplémentaires: la promotion de modèles et de comportements égalitaires ( 2 ententes : régions des Laurentides et du Bas-SaintLaurent), l'amélioration de la santé et de la sécurité des femmes (3 ententes : régions de la Mauricie, du Centre-du-Québec et du Bas-Saint-Laurent) en sont des exemples. Le territoire cri du Nord-du-Québec et la région de l'Outaouais font toutefois exception, leurs ententes incluant respectivement l'intégrité physique et la violence faite aux femmes, sans pourtant que des groupes de femmes n'en soient signataires. Trois régions ont choisi une formulation insensible au genre comme titre de leurs volets de l'entente : la région de la Côte-Nord favorise «les initiatives structurantes régionales-locales et [...] le maillage entre les différents intervenants du milieu », tandis que la région de Laval a opté pour la «revitalisation urbaine intégrée (RUI) » et la région de Longueuil a choisi le renforcement de ses instances de concertation.

Cinq des six orientations du plan d'action gouvernemental en matière d'égalité du MCCCF sont reflétées dans les volets de ces différentes ententes spécifiques régionales. L'égalité économique $(21 \text { ententes })^{14}$ et la participation des femmes aux instances (15 ententes) sont les plus fréquentes. Seule l'orientation portant sur la conciliation des responsabilités familiales et professionnelles est absente. La promotion de modèles et de comportements égalitaires apparaît dans 4 ententes, la thématique de la santé, dans 4 ententes, et la sécurité, dans 3 ententes. L'ADS (11 ententes) et la participation à la vie démocratique et citoyenneté (4 ententes) sont les thèmes les plus récurrents de volets d'ententes spécifiques qui ne correspondent pas précisément aux orientations du plan d'action gouvernemental en matière d'égalité. Considérant que l'ADS est une priorité transversale du plan d'action du MCCCF, on peut conclure avec le Vérificateur général que «très peu

13 Les volets les plus récurrents des ententes de la première génération étaient similaires : entrepreneuriat, représentation politique, emploi et qualité de vie (Masson 2004).

14 Nous avons regroupé sous ce vocable l'égalité économique (6 ententes), l'égalité en emploi ( 7 ententes) et l'entrepreneuriat au féminin ( 9 ententes). 
LE DÉFI DE L'ÉGALITÉ EN CONTEXTE DE DÉVELOPPEMENT RÉGIONAL | 107

d'ententes spécifiques ont donné lieu à une modulation des programmes » gouvernementaux pour prendre en considération «les besoins et priorités des communautés » (Gouvernement du Québec 2011 : 3-4).

La présence d'un ou de plusieurs groupes de femmes régionaux ou d'instances «femmes» à titre de signataires peut être corrélée avec le choix d'orientations du plan d'action du MCCCF les moins retenues par les CRE : la promotion de modèles et de comportements égalitaires (région des Laurentides) et l'amélioration de la santé et de la sécurité (région du Bas-Saint-Laurent).

La majorité des ententes spécifiques (12 sur 21) n'ont pas de groupe de femmes ni de table de concertation à titre de signataire. Plusieurs raisons peuvent expliquer cette situation, la principale étant bien sûr qu'il fallait, dans la plupart des cas, investir de l'argent sonnant dans l'entente spécifique pour pouvoir en être signataire. Il semble aussi que les groupes de femmes ou des tables de concertation qui auraient investi des sommes et ainsi accédé au comité décisionnel de leur entente spécifique interviennent tous dans les domaines jugés prioritaires par les ententes: développement économique, emploi, entrepreneuriat et représentation politique.

La faible présence des groupes de femmes ou des tables de concertation comme signataires confirme nos inquiétudes quant à la nature du rôle qui leur est réservé dans les ententes spécifiques. Rappelons que ces ententes concentrent le financement régional prévu en matière de condition féminine. Par la même occasion, elles limitent généralement ce financement aux projets harmonisés avec les priorités gouvernementales et exigent même souvent un investissement financier des groupes de femmes. On peut donc conclure que, dans sa facture actuelle, l'intégration des dossiers d'égalité au sein de la régionalisation québécoise correspond à une mise en œuvre du plan d'action du MCCCF. On peut donc toujours affirmer, comme le font Lamoureux et Pedneault (1999:1), que «les compétences des instances locales et régionales se limitent [...] à la mise en place de politiques [et] s'insèrent dans des orientations générales et des stratégies globales conçues ailleurs ».

Cela s'effectue paradoxalement, rappelons-le, sans balises claires transmises par le gouvernement du Québec aux autorités régionales, ce qui laisse cours à des interprétations multiples (Gouvernement du Québec 2011). Les initiatives locales des groupes de femmes régionaux se trouvent ainsi entièrement soumises aux décisions de conseils d'administration de CRE auxquels elles ne participent pas directement et qui interprètent très souvent à leur manière, la mise en œuvre des objectifs liés à l'atteinte de l'égalité sans avoir recours aux expertises pourtant présentes dans la région au sein des groupes de femmes. 
CÔTÉ ET TREMBLAY-FOURNIER $\mid 108$

\section{Conclusion}

L'« ouverture timide [...] des ministères et organismes [régionaux]» québécois aux groupes de femmes est chose du passé; les dossiers liés aux conditions de vie des femmes sont désormais partie prenante du développement régional au Québec. Ils sont intégrés et financés à travers les ententes spécifiques en matière d'égalité qui mobilisent des dispositifs régionaux (Minguy 2002:9) et formalisent ainsi les rapports avec les groupes de femmes de leur territoire.

Ces nouveaux dispositifs de gouvernance ont complètement reconfiguré l'espace social (St-Hilaire 1995) régional au sein duquel les groupes de femmes interviennent (Lamoureux et Pedneault 1999:25): «Le réseau des groupes de femmes [régionaux], particulièrement développé au Québec, [...] est confronté et intégré à une logique tout autre que la sienne, [...] le rendant de plus en plus dépendant de l'État, et l'intégrant toujours plus au "consensus". »

Pour le gouvernement québécois, les questions de l'égalité de la participation des groupes de femmes régionaux sont désormais assurées: la mobilisation conjuguée d'acteurs et de ressources régionales, dont les groupes de femmes, à travers ces ententes spécifiques, permettraient à leur point de vue une utilisation optimale des programmes existants et des budgets prévus sans investissement supplémentaire (Bourque 2009; Lamoureux 2005). Or en se limitant largement à la coordination de programmes existants, ces ententes spécifiques ne parviennent pas toutefois, dans la plupart des cas, à atteindre leur objectif de «moduler les programmes gouvernementaux en fonction des réalités régionales » (Gouvernement du Québec 2011 : 4).

De plus, l'inclusion des groupes de femmes régionaux dans ces nouveaux lieux décisionnels semble faire défaut ${ }^{15}$. Si les autorités régionales ne les ignorent plus ni ne les méconnaissent (Minguy 2002), leur expertise reste cependant reléguée à la mise en œuvre de plans d'action plutôt qu'à un rôle décisionnel. Absentes des conseils d'administration des CRE ou des comités directeurs des ententes spécifiques, la grande majorité des groupes de femmes ou des tables de concertation régionale actifs dans les ententes spécifiques occupent ainsi une niche de soustraitant privilégié.

La reconnaissance des groupes de femmes régionaux n'est donc que partielle en ce sens qu'elle ne leur donne qu'un accès limité aux instances et ne garantit en rien le financement de projets endogènes non liés aux priorités gouvernementales. Par la même occasion, le bénévolat exigé par les processus de concertation des CRE connaît une augmentation exponentielle. Le financement par projet se généralise, de même que la sous-traitance et l'approche de gestion de

15 Les groupes de femmes n'auront jamais la possibilité d'exercer un pouvoir décisionnel au sein des ententes spécifiques si la qualité requise pour en devenir signataire, et donc décideur, demeure celle d'un versement initial de liquidités à ladite entente. 
LE DÉFI DE L'ÉGALITÉ EN CONTEXTE DE DÉVELOPPEMENT RÉGIONAL | 109

projet dont l'efficacité dans la sphère des droits de la personne reste à être démontrée. Cette contractualisation des rapports force l'adoption, au sein des groupes de femmes, de la logique de concertation propre aux CRE ainsi que de la logique administrative gouvernementale. Tout au moins doivent-ils concilier constamment leur logique communautaire avec celles-ci.

Se crée ainsi parallèlement un marché régional de consultation dans le « champ de l'égalité » auquel peuvent s'associer les groupes de femmes. Une culture et des pratiques de sous-traitance s'introduisent par ce biais au sein de la culture communautaire et dans le fonctionnement des groupes: les groupes de femmes « coûtent» moins cher que les services publics qui les sollicitent, " [leurs] employés ne jouissant pas des salaires et des avantages sociaux des travailleurs des secteurs public et parapublic » (Piotte $2010: 8$ ).

Ces groupes sont alors mis en concurrence avec le secteur privé de la consultation. Et dans ce nouvel espace qui leur est réservé, les groupes de femmes ont bien souvent de la difficulté à faire valoir leur vision communautaire et féministe.

La décentralisation n'aura donc pas permis de répondre à l'aspiration des groupes de femmes pour une société civile régionale qui serait un «lieu d'une horizontalité reflétant [une] volonté démocratique" (Lamoureux et Pedneault 1999 : 41). Les ententes spécifiques en matière d'égalité ne sont pas conçues pour combler un tel déficit démocratique. Dans la plupart des cas, elles n'auront pas été l'occasion " pour l'échange [souhaité] d'idées entre citoyennes et citoyens » sur des problèmes sociaux. Elles seront plutôt devenues un forum de " rencontres d'intérêts, de groupes d'intérêts, [qui occasionnent] de nombreuses luttes corporatistes autour des organisations régionales » (Minguy 2002 : 10). Et l'intensification des maillages entre acteurs nourrira paradoxalement le déficit démocratique régional. Déjà, les CRE ne reconnaissent qu'une certaine expression institutionnelle du mouvement féministe régional qui, on le sait, ne s'y limite pas (Eschle 2001).

Ces ententes spécifiques encouragent-elles la mise en veilleuse de la mission originale de défense des droits qui reste celle des groupes de femmes (Falquet 2008; Lamoureux et Pedneault 1999; Marques-Pereira 2000)? Créent-elles les conditions au Québec, comme dans certains cas rapportés en Europe et dans le Sud (MarquesPereira 2007; Jouve 2005) "pour coopter une partie de ce mouvement [social]» (Biel 2003 : 31)? Et dans quelle mesure la nouvelle architecture régionale prend-elle en considération les droits fondamentaux des femmes ou coopte-t-elle ces droits (Brunelle 2010)? Car ce rapprochement des autorités régionales et de la société civile ouvre toute grande la voie au «rapt» par les élites locales de projets, de discours et de dossiers issus de la base (Lebovics 2007).

Les intérêts et les rapports de pouvoirs au sein des régions restent diversifiés, pluralistes et liés aux niveaux national et international (Eschle 2001). D'ailleurs, selon Labrecque (1997:34), on assiste trop souvent encore à la « négation de la complexité des rapports sociaux [car] les méthodologies utilisées 
CÔTÉ ET TREMBLAY-FOURNIER $\mid 110$

par les praticiens du développement permettent difficilement d'aborder les rapports entre les hommes et les femmes comme des processus dynamiques de changement social ».

En effet, les nouvelles modalités de gouvernance régionale semblent réserver aux groupes de femmes un rôle d'influence et de mise en œuvre d'orientations gouvernementales articulées au gré des priorités d'élites régionales plutôt qu'une occasion de défendre les droits des femmes en participant pleinement aux processus décisionnels.

Enfin, rappelons que les cadres d'interprétation des nouvelles structures de gouvernance font cruellement défaut au Québec (Tremblay, Tremblay et Tremblay 2002). L'articulation des rapports entre la société civile, les associations qui en sont issues et les structures de gouvernance méritent aussi une analyse plus poussée : tout système démocratique repose en effet sur une société civile forte et autonome. À ce jour, l'État reste encore le seul dispositif capable de définir le bien commun et d'élaborer les cadres de mise en œuvre des droits de ses citoyens. Il est toujours de sa responsabilité de définir le cadre du financement et des échanges de services avec le monde associatif, qui ne pourra se réaliser qu'à partir d'un dialogue horizontal entre ce dernier et l'instance gouvernementale visée sur les objectifs et les moyens à privilégier.

\section{RÉFÉRENCES}

ASHWORTH, Georgina

1996 Gendered Governance : An Agenda for Change. New York, United Nations Development Programme.

BALLMER-CAO, Thanh-Huyen

2006 «Genre et régulation », dans Lorena Parini, Thanh-Huyen Ballmer-Cao et Sylvie Durrer, Régulation sociale et genre. Paris, L'Harmattan : 15-30.

BARON, Catherine

2007 «Transfert du concept d'économie solidaire en Afrique francophone», Revue Tiers-Monde, 2, $190: 325-334$.

BIEL, Robert

2003 «Le capitalisme a besoin des femmes », dans Jeanne Bisilliat (dir.), Regards de femmes sur la globalisation. Paris, Karthala : 27-34.

BISILLIAT Jeanne

2000 «Luttes féministes et développement: une perspective historique », dans Jeanne Bisilliat et Christine Verschuur (dir.), Le genre : un outil nécessaire, introduction à une problématique. Paris, Cahiers genre et développement : 19-29.

BOURQUE, Denis

2009 Concertation et partenariat. Québec, Presses de l'Université du Québec. 
LE DÉFI DE L'ÉGALITÉ EN CONTEXTE DE DÉVELOPPEMENT RÉGIONAL | 111

BRUNELLE, Dorval

2010 Gouvernance théorie et pratiques. Montréal, Éditions IEIM.

2009 L'autre société civile, les mouvements sociaux et la lutte pour les droits fondamentaux. Québec, Les Presses de l'Université Laval.

CARRASCO, Diego

2000 "Contribution of the Plataforma continental de derechos humanos to the Open Invitation "), Free Trade Area of the Americas, Committee of Government Representatives on the Participation of Civil Society. [En ligne], [www.ftaa-alca.org/spcomm/soc/Contributions/BAires/ csw151a1_e.asp] (février).

CENTRE DE DOC̄UMENTATION SUR L'ÉDUCATION DES ADULTES ET LA

CONDITION FÉMININE (CDEACF)

2011 Cartographie des groupes de femmes. [En ligne], [netfemmes.cdeacf.ca/les_ressources/bottin/index.php] (février).

CHEVALIER, Jacques

2003 «La gouvernance, un nouveau paradigme étatique?», Revue française d'administration publique, 1-2, 105-106 : 203-217.

CÔTÉ, Denyse

1995 «Développement communautaire et rapports sociaux de sexe: essai d'analyse sur toile de fond régionale », dans Denyse Côté et autres (dir.), $D u$ local au planétaire, Réflexions et pratiques de femmes en développement régional. Montréal, Les éditions du remue-ménage : 109-133.

CÔTÉ, Denyse et Étienne SIMARD

2010 «De l'utopie radicale à la bonne gouvernance: le cas du Québec», AmeriQuests, 7, $1: 42-53$.

DEGRAVE, Florence

2000 «Les différents courants de pensée intégrant "femmes" et "développement" ", Chronique féministe, 71-72, mai : 80-82.

DUTRISAC, Robert

2011 «Les groupes de femmes en rogne contre St-Pierre », Le Devoir, 8 avril.

ESCHLE, Catherine

2001 Global Democracy, Social Movements and Feminism. Boulder, CO, Westview Press.

FALQUET, Jules

2008 De gré ou de force. Les femmes dans la mondialisation. Paris, La Dispute.

FRASER, Nancy

1989 Unruly Practices : Power, Discourse, and Gender in Contemporary Social Theory. Minneapolis, University of Minnesota Press.

GOUVERNEMENT DU QUÉBEC (ASSEMBLÉE NATIONALE)

2003 Loi 34 créant le ministère du Développement économique et régional. Québec, Éditeur officiel. 
CÔTÉ ET TREMBLAY-FOURNIER | 112

GOUVERNEMENT DU QUÉBEC (SECRÉTARIAT À LA CONDITION FÉMININE)

2007 Pour qu'une égalité de droit devienne une égalité de fait, Plan d'action 2007-2010. Québec, Éditeur officiel.

1997a Cinquième orientation: La place des femmes dans le développement régional. Québec, Éditeur officiel.

1997b Un avenir à partager, la politique en matière de condition féminine: programme d'action 1997-2000 pour toutes les Québécoises. Québec, Éditeur officiel.

GOUVERNEMENT DU QUÉBEC (VÉRIFICATEUR GÉNÉRAL)

2011 Rapport du Vérificateur général à l'Assemblée nationale, chapitre 4: interventions en matière de développement régional. Québec, Éditeur officiel.

GRAEFE, Peter

2001 «Whose Social Economy? Debating New State Practices in Québec», Critical Social Policy, 21, 25 : 34-58.

HONNETH, Axel

2008 La société du mépris. Paris, La Découverte.

JAGLIN, Sylvy

2005 «La participation au service du néolibéralisme? Les usagers dans les services d'eau en Afrique subsaharienne », dans Marie-Hélène Bacqué, Henri Rey et Yves Syntomer (dir.), Gestion de proximité et démocratie participative : une perspective comparative. Paris, La Découverte : 271-329.

JOUVE, Bernard

2005 «La démocratie en métropoles : gouvernance, participation et citoyenneté », Revue française de science politique, 55, 2, avril : 317-337.

LABRECQUE, Marie France

1997 Sortir du labyrinthe. Femmes, développement et vie quotidienne en Colombie andine. Ottawa, Presses de l'Université d'Ottawa.

LAFONTAINE, Danièle et Nicole THIVIERGE

1997 «Les femmes actrices de changement et le devenir des espaces urbains et ruraux régionaux au Québec », Recherches féministes, 10, 2 : 137-153.

LAMOUREUX, Diane

2005 «Les tentatives d'instrumentalisation de la société civile par l'État», dans Francine Saillant et Éric Gagnon (dir.), Communautés et socialités. Montréal, Liber : 39-58.

LAMOUREUX, Diane et Évelyne PEDNEAULT

1999 Les femmes et les enjeux démocratiques locaux et régionaux: rapport de recherche. Québec, Chaire d'étude Claire-Bonenfant sur la condition des femmes, Université Laval. 
LE DÉFI DE L’ÉGALITÉ EN CONTEXTE DE DÉVELOPPEMENT RÉGIONAL | 113

LAURIN-FRENETTE, Nicole

1999 « Le démantèlement des institutions intermédiaires de la régulation sociale. Vers une nouvelle forme de domination », Sociologie et sociétés, 31, 2, automne : 65-72.

1984 «Féminisme et anarchisme : quelques éléments théoriques et historiques pour une analyse de la relation entre le mouvement des femmes et l'État », dans Nicole Laurin-Frenette, Yolande Cohen et Kathy Ferguson (dir.), Femmes: pouvoir, politique, bureaucratie. Lyon, Atelier de création libertaire : $13-55$.

LEBOVICS, Maxime

2007 " Analyse des apports et des contraintes du développement participatif», Afrique contemporaine, 3-4, 223-224 : 403-432.

LEMIEUX, Vincent

1997 La décentralisation. Sainte-Foy, Les Éditions de l'Institut québécois de recherche sur la culture (IQRC), Les Presses de l'Université Laval.

MARQUES-PEREIRA, Bérengère

2007 «Le Chili et les rapports entre les sexes. Quel "régime de citoyenneté » pour les femmes? », dans Jane Jenson et autres (dir.), L'État des citoyennetés en Europe et dans les Amériques. Montréal, Les Presses de l'Université de Montréal : 81-100.

2000 "Gouvernance, citoyenneté et genre », dans Corrine Gobin et Benoît Rihoux (dir.), La démocratie dans tous ses états. Louvain-la-Neuve, Bruylant-Academia : 41-50.

MASSOLO, Alejandra

2000 «La incursión de las mujeres en los procesos de descentralización », dans Corporación de estudios para el desarrollo, Mujer, Participación y Desarrollo. Quito, Corporación de estudios para el desarrollo (CORDES) / Centro para la investigación y el desarrollo de los movimientos sociales del Ecuador (CEDIME) : 1-31.

MASSON, Dominique

2006 «Femmes, gouvernance régionale et enjeux de citoyenneté », dans Martin Robitaille, Jean-François Simard et Guy Chiasson (dir.), L'Outaouais au carrefour des modèles de développement. Gatineau, Université du Québec en Outaouais, Centre de recherche sur le développement territorial (CRDT) et Chaire de recherche sur le développement communautaire (CRDC) : 145158.

2004 «Formation des discours féministes et action institutionnelle : l'articulation des intérêts des femmes dans le développement régional au Québec », Labrys études féministes, 6, août/décembre, n. p.

2001 «Gouvernance partagée, associations et démocratie: les femmes dans le développement régional », Politique et Sociétés, 20, 2-3 : 89-115. 
CÔTÉ ET TREMBLAY-FOURNIER $\mid 114$

MINGUY, Claire

2002 « Nouveaux enjeux de la participation des femmes aux lieux décisionnels dans le contexte de la régionalisation », dans Nicole Thivierge et Marielle Tremblay, Régionalisation et démocratie : les défis d'une citoyenneté active pour les femmes. Montréal, Réseau féministe de chercheuses et d'intervenantes pour un renouvellement des théories et des pratiques économiques et politiques pour la redistribution des richesses : 9-15.

PECK, Jamie

2004 « Geography and Public Policy : Constructions of Neoliberalism », Progress in Human Geography, 28, 3 : 392-405.

PIOTTE, Jean-Marc (sous la direction du Comité gouvernance de l'État)

2010 L'État social et l'action communautaire autonome. Montréal, Réseau québécois d'action communautaire autonome.

RATHGEBER, Eva

1995 «Gender and Development in Action », dans Marianne H. Marchand et Jane L. Parpart (dir.), Feminism, Postmodernism, Development. Londres et New York, Routledge : 204-220.

RIST, Gilbert

1996 Le développement. Histoire d'une croyance occidentale. Paris, Presses de Sciences Po.

SASSEN, Saskia

2009 La globalisation, une sociologie. Paris, Gallimard.

ST-HILAIRE, Colette

1995 «Études féministes et paradigmes du développement : de l'interpellation à l'articulation ", dans Denyse Côté et autres (dir.), Du local au planétaire, réflexions et pratiques de femmes en développement régional. Montréal, Les éditions du remue-ménage : 17-41.

TARDY, Évelyne

2002 Les femmes et les conseils municipaux du Québec. Montréal, Éditions Hurtubise.

TREMBLAY, Marielle, Pierre-André TREMBLAY et Suzanne TREMBLAY (dir.)

2002 Développement local, économie sociale et démocratie. Québec, Presses de l’Université du Québec. 